\title{
Accuracy and Stability of 2D-XRD for Residual Stress Measurement
}

\author{
Bob B. He* \\ 5465 East Cheryl Parkway, Madison, WI 53711, USA \\ bob.he@bruker.com
}

\section{Keywords: Residual Stress, 2D Detector, Accuracy, Stability}

\begin{abstract}
Stress measurement with two-dimensional X-ray diffraction is based on the direct relationship between the stress tensor and diffraction cone distortion. Since more data points at more orientations are used for stress calculation, 2D-XRD can measure stress with high sensitivity, high speed and high accuracy. It is especially suitable for samples with large crystals, textures and curved surface. Ten almen strips were used for Gage R\&R test of a 2D-XRD system for stress measurement in 1998. Since then there have been many advances in two-dimensional X-ray diffractometer, including detector technology, X-ray source and optics, goniometer, and data evaluation software. This paper covers accuracy and stability of stress measurement on the same almen strips with a 2DXRD system.
\end{abstract}

\section{Introduction}

Two-dimensional x-ray diffraction (abbreviated as $\mathrm{XRD}^{2}$ ) pattern contains information in a large solid angle which can be described by the diffraction intensity distribution in both $2 \theta$ and $\gamma$ directions [1]. The $2 \theta$ peak position can be determined from each section of the diffraction ring within a $\gamma$ range. The $\mathrm{d}$-spacing variation at different orientations due to stress results in $2 \theta$ peak position variation at different $\gamma$ angle and sample orientation. For a particular sample orientation, the variation of $2 \theta$ along $\gamma$ angle is observed as the distortion of the diffraction ring on $2 \mathrm{D}$ diffraction pattern. A set of $2 \mathrm{D}$ diffraction patterns collected at various sample orientations can be used to evaluate the stress tensor in the sample. Since each 2D diffraction pattern covers one or several diffraction rings with large $\gamma$ range, the $\mathrm{XRD}^{2}$ method can measure stress with high accuracy and high speed, especially when dealing with texture, large grain size, small sample areas and curved sample surface [2-5].

The accuracy of stress measurement by $\mathrm{XRD}^{2}$ system depends on many factors, such as, the spherical error of the goniometer, sample position, the detector, profile fitting algorithms and data collection strategy. Gage repeatability and reproducibility (Gage R\&R) test on residual stress measurement with an $\mathrm{XRD}^{2}$ system was reported in 1998 [6]. The test was performed by three operators with three measurements on 10 almen strips. The test shows that the overall measurement error is $6 \%$ or less. The repeatability error of the system is $5.5 \%$ or less, and the reproducibility error is $2.3 \%$ or less. The average stress for all 90 measurements is $-628 \pm 19 \mathrm{MPa}(3.1 \%)$. The sample from the same set of almen strips has been used to evaluate many $\mathrm{XRD}^{2}$ systems in terms of the accuracy and system stability in the past 18 years. This paper covers accuracy and stability of stress measurement with an $\mathrm{XRD}^{2}$ system containing recent advances in X-ray source, goniometer and detector.

\section{Fundamental Equation for Stress Measurement}

Fig. 1 illustrates a diffraction cone for backward diffraction. The regular diffraction cones (broken lines) are from a sample with no stress, so the $2 \theta$ angles are constant at all $\gamma$ angles. The solid line is the cross sections of the distorted diffraction cone due to stresses. For a stressed sample, $2 \theta$ becomes 
a function of $\gamma$ and the sample orientation $(\omega, \psi, \phi)$. The fundamental equation for stress measurement with $\mathrm{XRD}^{2}$ can be given as [3]:

$$
\boldsymbol{S}_{1}\left(\sigma_{11}+\sigma_{22}+\sigma_{33}\right)+\frac{1}{2} \boldsymbol{S}_{2}\left(\sigma_{11} \boldsymbol{h}_{1}^{2}+\sigma_{22} \boldsymbol{h}_{2}^{2}+\sigma_{33} \boldsymbol{h}_{3}^{2}+2 \sigma_{12} \boldsymbol{h}_{1} \boldsymbol{h}_{2}+2 \sigma_{13} \boldsymbol{h}_{1} \boldsymbol{h}_{3}+2 \sigma_{23} \boldsymbol{h}_{2} \boldsymbol{h}_{3}\right)=\ln \left(\frac{\sin \theta_{0}}{\sin \theta}\right)
$$

where $\boldsymbol{S}_{1}$ and $\frac{1}{2} \boldsymbol{S}_{2}$ are the macroscopic elastic constants, and $\boldsymbol{h}_{\mathbf{1}}, \boldsymbol{h}_{\mathbf{2}}$ and $\boldsymbol{h}_{\mathbf{3}}$ are the three components of the unit diffraction vector expressed in sample coordinates. For Eulerian geometry:

$\boldsymbol{h}_{1}=\sin \theta(\sin \phi \sin \psi \sin \omega+\cos \phi \cos \omega)+\cos \theta \cos \gamma \sin \phi \cos \psi-\cos \theta \sin \gamma(\sin \phi \sin \psi \cos \omega-\cos \phi \sin \omega)$

$\boldsymbol{h}_{2}=-\sin \theta(\cos \phi \sin \psi \sin \omega-\sin \phi \cos \omega)-\cos \theta \cos \gamma \cos \phi \cos \psi+\cos \theta \sin \gamma(\cos \phi \sin \psi \cos \omega+\sin \phi \sin \omega)$

$\boldsymbol{h}_{3}=\sin \theta \cos \psi \sin \omega-\cos \theta \sin \gamma \cos \psi \cos \omega-\cos \theta \cos \gamma \sin \psi$

Eq. 1 can be further reduced for various stress states and instrument conditions. For example, if only the data points at $\gamma=90^{\circ}$ or $\gamma=-90^{\circ}$ are considered, the equation can be reduced to the fundamental equation for stress measurement with point detector [1]. For most materials, X-ray diffraction can measure only a very thin layer on the surface, it is reasonable to assume that the average normal stress in the surface normal direction is zero within such a thin layer, i.e. $\sigma_{33}=0$. In this case, the other five components of the stress tensor can be measured without accurate stress-free d-spacing.

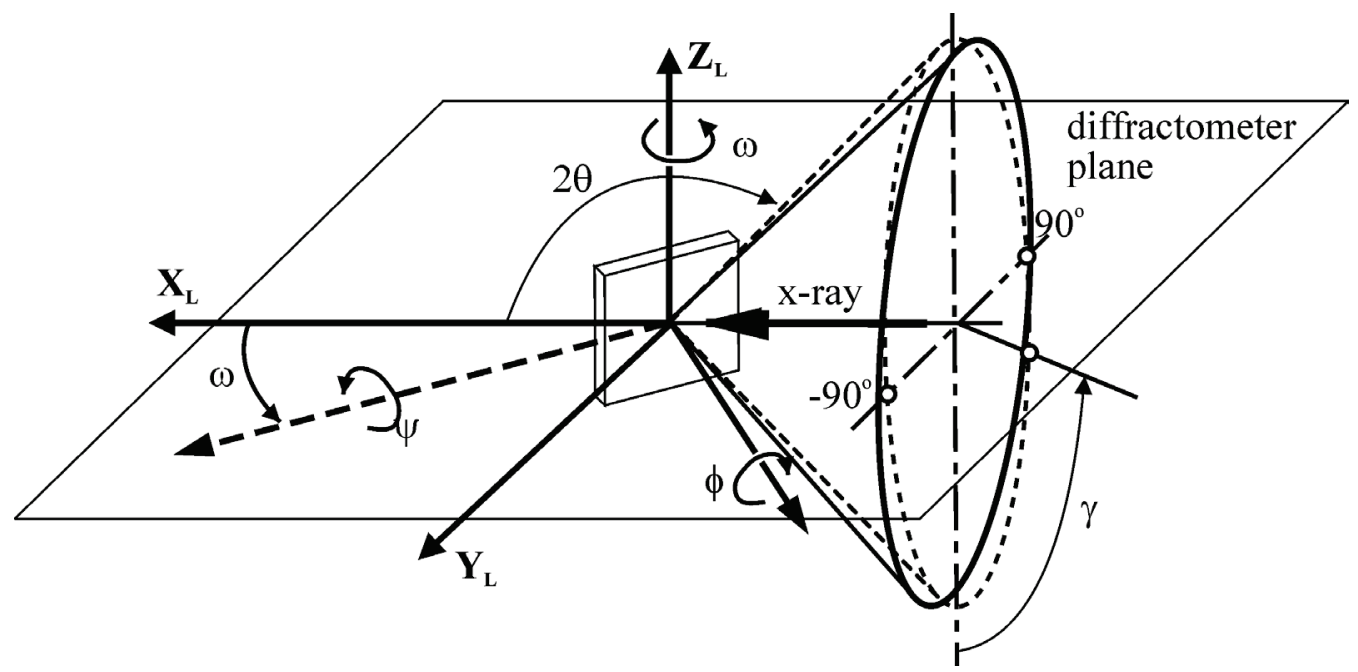

Fig. 1. Sample orientation and diffraction cone distortion due to stress.

\section{Instrumentation and Data Collection Strategy}

The $\mathrm{XRD}^{2}$ system used for the experiment is Bruker D8 DISCOVER diffractometer. As shown in Fig. 2, it contains I $\mu \mathrm{S}$ microsource X-ray generator with $\mathrm{Cr}-\mathrm{K} \alpha$ radiation, VÅNTEC-500 2D detector, Eulerian cradle, dual lasers and video microscope. The $2 \theta$ of $\mathrm{Fe}$ (211) peak with $\mathrm{Cr} \mathrm{K} \alpha$ radiation is approximately $156^{\circ}$, so the incident angle is set at $\theta_{1}(\omega)=78^{\circ}$. The detector is set at $\theta_{2}=71^{\circ}$ (swing angle $\alpha=149^{\circ}$ ) at sample-to-detector distance $234 \mathrm{~mm}$. The diffraction vector direction is determined by the incident angle and the $2 \mathrm{D}$ detector covers about $30^{\circ} 2 \theta$ range, which is sufficient to cover the (211) ring with complete background. The collimator size is $0.5 \mathrm{~mm}$. The cross point of the dual laser beam indicates the instrument center which can be observed with the video microscope. The motorized $\mathrm{X}-\mathrm{Y}-\mathrm{Z}$ stage on the Eulerian cradle allows precise position of the sample. The generator power setting in this experiment is $15 \mathrm{~W}(40 \mathrm{kV} / 0.375 \mathrm{~mA})$. 


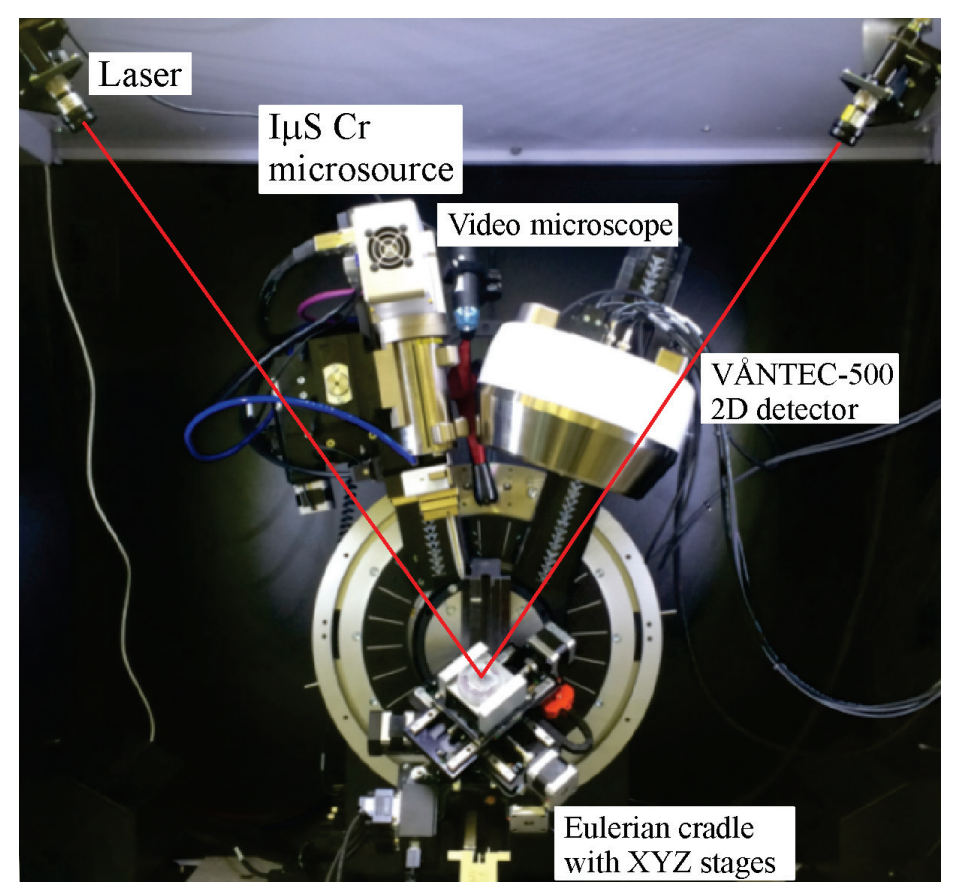

Fig. 2. The XRD $D^{2}$ diffractometer used for the experiment: D8 DISCOVER with $\mathrm{Cr} I \mu S$ microsource, VANTEC-500, and Eulerian cradle.

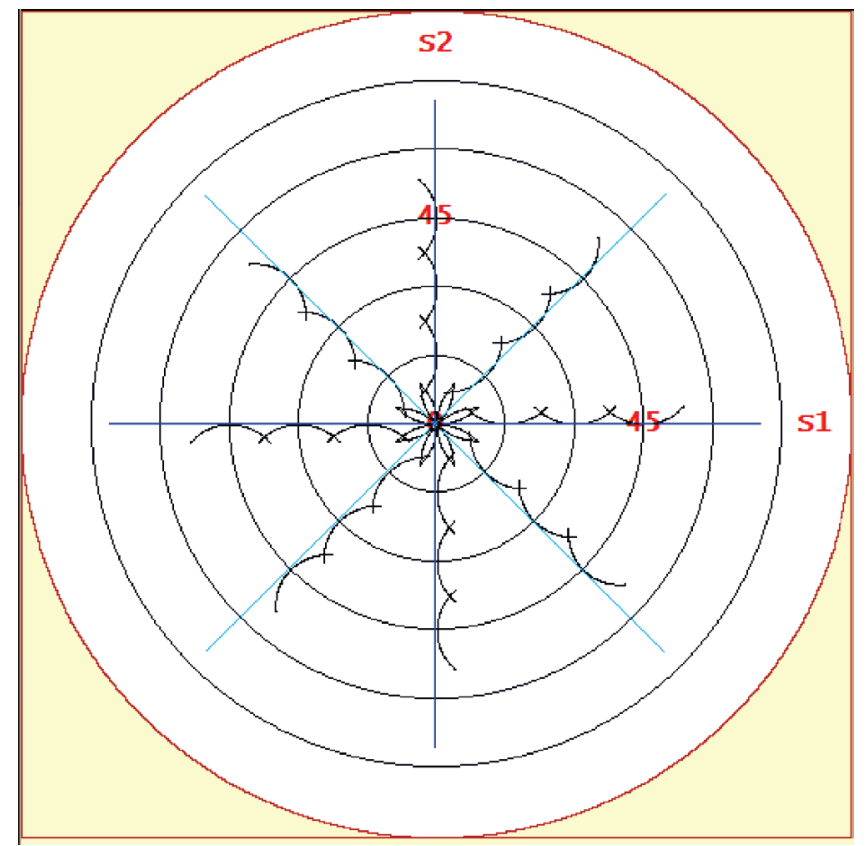

Fig. 3. Data collection strategy schemes with 32 frames at $\psi=0,15^{\circ}, 30^{\circ}, 45^{\circ}$ with complete $\phi$ rotation of $45^{\circ}$ steps.

The diffraction vector is in the normal direction of the measured crystalline planes. It is not always possible to have the diffraction vector in the desired measurement direction. The stress components within the surface plane are calculated by elasticity theory from the measured strain in other directions. The final stress measurement results can be considered as an extrapolation from the measured values. For example, in the conventional $\sin ^{2} \psi$ method, the stress in the sample surface 
$\left(\psi=90^{\circ}\right)$ is calculated from the measured strains at several $\psi$-tilt angles. The same is true with an $\mathrm{XRD}^{2}$ method. Similar to a pole-figure, the diffraction vectors corresponding to a data set can be mapped in a data collection strategy scheme. By evaluating the scheme, one can collect a data set optimized for the intended stress components. Fig. 3 illustrates the scheme used for data collection with this experiment. The small arcs represent the trace of the diffraction vector corresponding to the data set. S1 and S2 are two sample orientations. In this scheme, 32 frames are collected at $\psi=0^{\circ}, 15^{\circ}$, $30^{\circ}$ and $45^{\circ}$ at eight $\phi$ angles with $45^{\circ}$ intervals. This scheme produces comprehensive coverage on the scheme chart in a symmetric distribution. The data set collected with this strategy can be used to calculate the complete biaxial stress tensor components and shear stress $\left(\sigma_{11}, \sigma_{12}, \sigma_{22}, \sigma_{13}, \sigma_{23}\right)$. A proper scheme is selected based on the interested stress components, the goniometer, desired measurement accuracy and data collection time.

\section{Stress Analysis}

The stress calculation is done with Bruker DIFFRAC.LEPTOS software version 7.9. Fig. 4 shows the data evaluation setting. The data integration region is defined by $2 \theta$ range of $150^{\circ}$ to $160^{\circ}$ and $\gamma$ range of $-70^{\circ}$ to $-110^{\circ}$. The $40^{\circ} \gamma$ range is divided into 8 subregions, $5^{\circ}$ for each subregion. The counts within each subregion are integrated into a diffraction profile and the $2 \theta$ peak position is determined by one of the five peak evaluation algorithms. In this experiment, Pearson VII function is used to fit the profile and evaluate the $2 \theta$ peak position. Fig. 5 shows the stress results from one of the data set. The charts above "A" are the fitted data points on 2D frames. The charts above " $\mathrm{B}$ " are fitted data points in $\gamma-2 \theta$ rectangular coordinates with magnified $2 \theta$ scale, in which, black line indicates $2 \theta_{0}$, blue cross and line indicates the data points from the profile fitting of each subregion, and red line represents the calculated diffraction rings from the stress results. The scattering of the crosses about the red line represents the quality of the data, affecting the standard deviation of the stress results. By click on any data point, the integrated profile displays above "C".

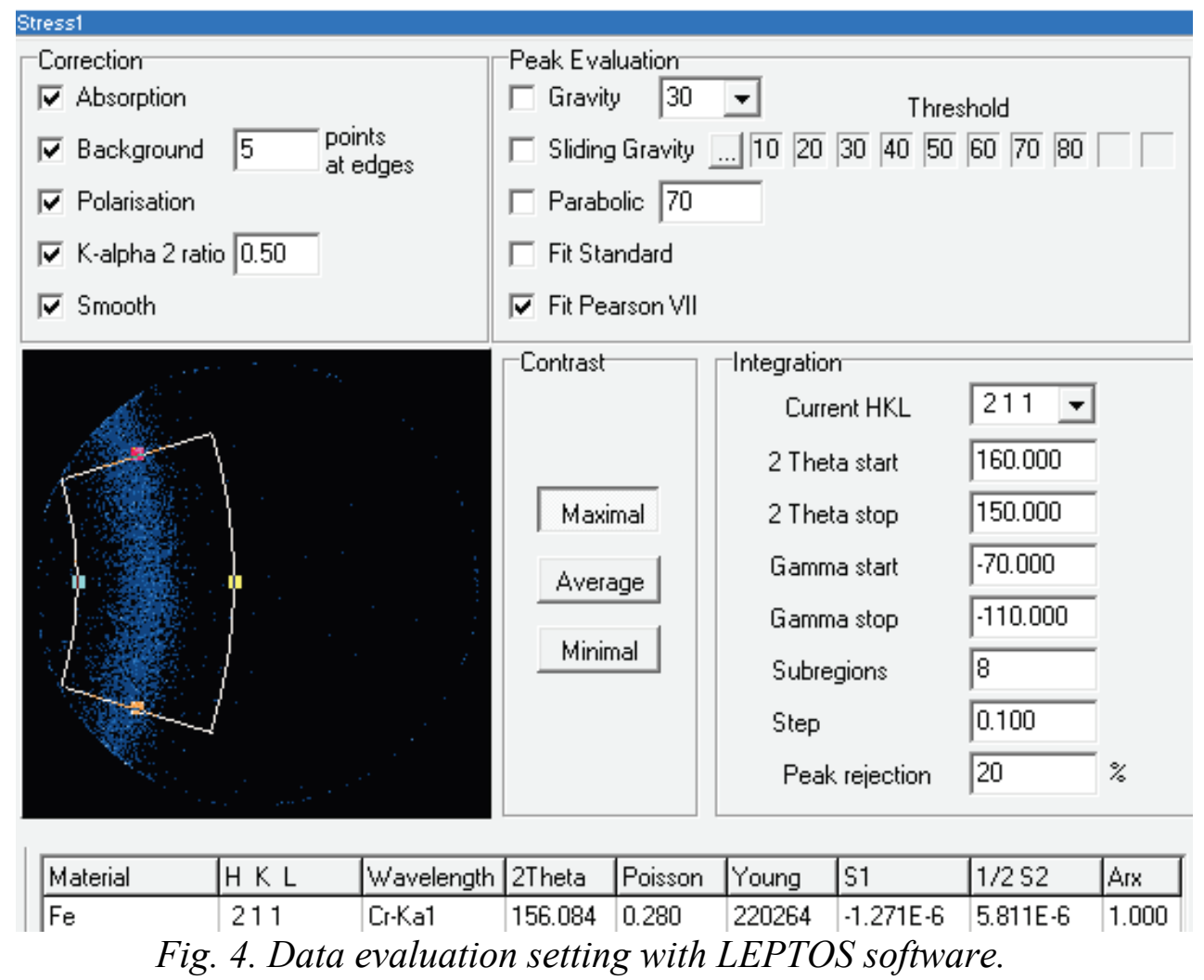




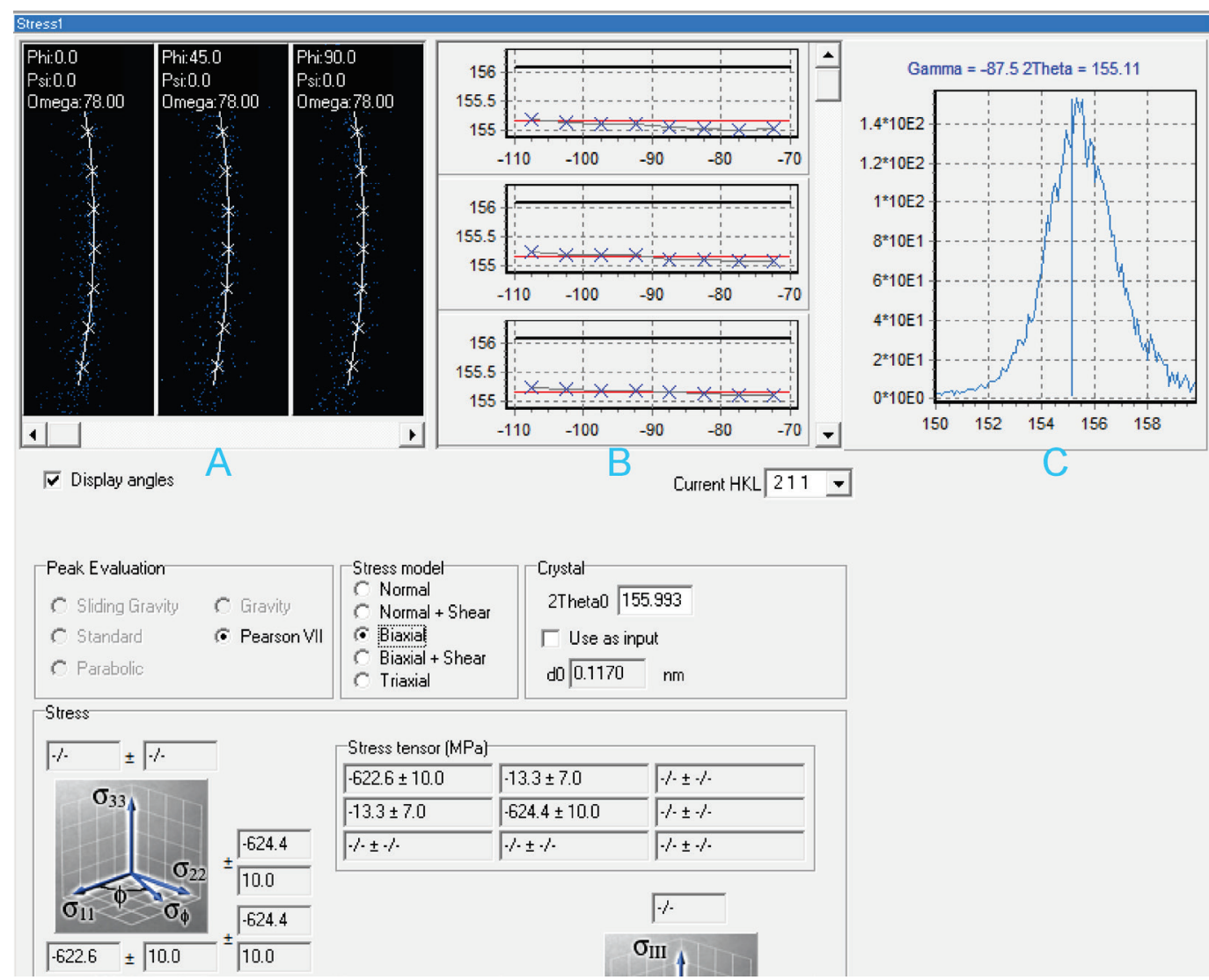

Fig. 5. Data evaluation results with LEPTOS software.

\section{Accuracy and Stability}

The stress-free Fe powder sample is used to check the instrument accuracy. Any spherical confusion of the goniometer and sample position error produces a fictitious stress value. With 60 second frames, the measured stress values are: $\sigma_{11}=13 \mathrm{MPa}, \sigma_{22}=9 \mathrm{MPa}$ and standard deviation $12 \mathrm{MPa}$. With 180 second frames, the measured stress values are: $\sigma_{11}=8 \mathrm{MPa}, \sigma_{22}=10 \mathrm{MPa}$ and standard deviation $9 \mathrm{MPa}$. The results satisfy the accuracy specification for the D8 Discover diffractometer.

Stability of the instrument is tested with one of the almen strips over 65 hours, with data collection of 120 second and 600 second per frame data set alternatively for 10 times. Fig. 6 shows the measurement results over the data collection time. Stress values from 600s frames are more stable compared with the results from 120s frames. The average values with $120 \mathrm{~s}$ frames are: $\sigma_{11}=-682$ $\mathrm{MPa}, \sigma_{22}=-664 \mathrm{MPa}$ with standard deviation $20 \mathrm{MPa}$. With 600 second frames, the average stress values are: $\sigma_{11}=-625 \mathrm{MPa}, \sigma_{22}=-616 \mathrm{MPa}$ with standard deviation $10 \mathrm{MPa}(1.6 \%)$. The $600 \mathrm{~s}$ results seem to be more consistent with the average value of $628 \mathrm{MPa}$ measured 18 years ago, assuming there is no stress relaxation in the almen strip. The results from the 120 s frames have twice the standard deviation and more systematic error compared with the results from $600 \mathrm{~s}$ frames.

Since the 120 s data set and 600 s data set are collected alternatively, the discrepancy is not likely due to the motion accuracy of the instrument, but most likely from the counting statistics. One of each integrated profiles are displayed in Fig. 6. It can be seen that the 600s profile is much smoother than the 120 s profile. In addition to the data collection time, there are many ways to improve the counting statistics, for instance, more powerful X-ray source or generator setting, large collimator size, and short source to sample distance. For instance, the $100 \mathrm{~mm}$ gap between the collimator and sample can be significantly reduced. As previously reported with a different system, the results from a data set of 5 second frames have less than $2 \%$ standard error [3]. 


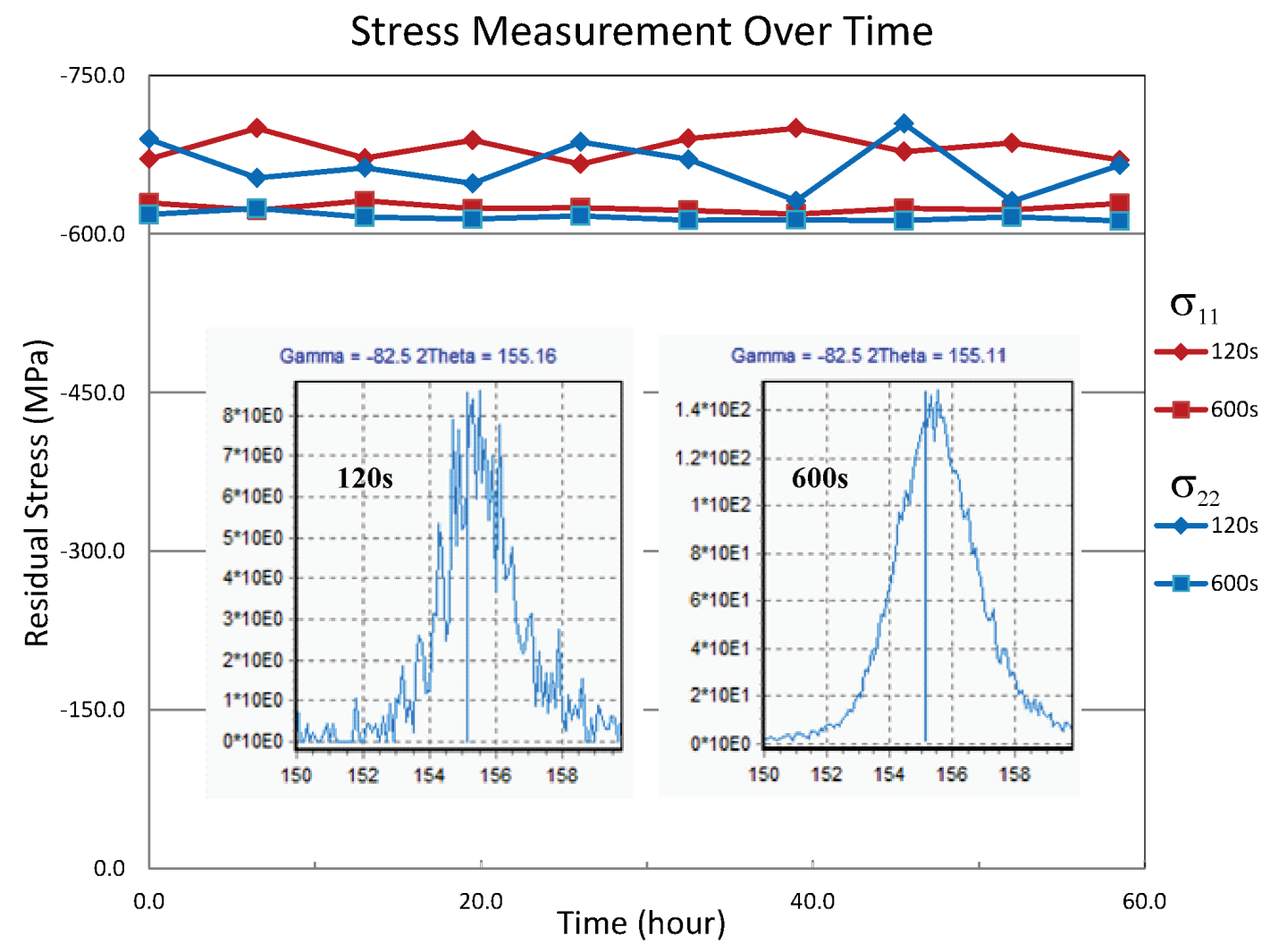

Fig. 6. Stress measurement results over time.

\section{Summary}

The $\mathrm{XRD}^{2}$ system can measure residual stress with high accuracy and stability. The sample position is maintained during repeatable data collection with high accuracy and stability. The stress measurement accuracy in terms of standard deviation and systematic error is mostly due to counting statistics. The residual stresses in the almen strips did not show a detectable change over 18 years.

\section{References}

[1] B. B. He, Two-dimensional X-ray Diffraction, John Wiley \& Sons, Hoboken, New Jersey 2009. http://dx.doi.org/10.1002/9780470502648

[2] B. B. He, U. Preckwinkel and K. L. Smith, Advantages of Using 2D Detectors for Residual Stress Measurement, Advances in X-ray Analysis, Vol. 42 (1998) pp. 429-438.

[3] B. B. He, MicroGap area detector for stress and texture analysis, Materials Science Forum, Vol. 681 (2011), pp. 19-24. http://dx.doi.org/10.4028/www.scientific.net/MSF.681.19

[4] B. B. He, XRD ${ }^{2}$ stress measurement for samples with texture and large grains, Materials Science Forum, Vols. 768-769 (2014) pp. 227-234. http://dx.doi.org/10.4028/www.scientific.net/MSF.768769.227

[5] T. Miyazaki and T. Sasaki, A comparison of X-ray stress measurement methods based on the fundamental equation, J. Appl. Cryst. Vol. 49 (2016) pp. 426-432. http://dx.doi.org/10.1107/S1600576716000492

[6] B. B. He, K. L. Smith, U. Preckwinkel and W. Schultz, Gage R\&R study on residual stress measurement system with area detector, Materials Science Forum Vols. 347-349 (2000) pp. 101-106. http://dx.doi.org/10.4028/www.scientific.net/MSF.347-349.101 